\title{
Author Correction: Selective synaptic remodeling of amygdalocortical connections associated with fear memory
}

Yang Yang, Dan-qian Liu, Wei Huang, Juan Deng, Yangang Sun, Yi Zuo and Mu-ming Poo

Correction to: Nature Neuroscience https://doi.org/10.1038/nn.4370, published online 5 September 2016.

In the version of this article initially published, Fig. $7 f$ purported to show an example of a multi-synapse spine. However, the structure in question included a mitochondrion and microtubules, meaning that it was actually a segment of dendritic shaft. A new image showing an example of a bona fide spine has been substituted. The legend has been changed to state that the image shows two boutons rather than three. The error has been corrected in the HTML and PDF versions of the article.
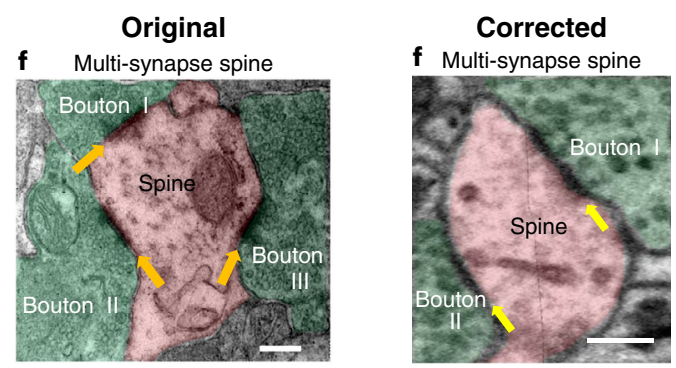

Fig. 7f | Original and corrected.

\section{Author Correction: Microglial immune checkpoint mechanisms}

Aleksandra Deczkowska (D), Ido Amit and Michal Schwartz

Correction to: Nature Neuroscience https://doi.org/10.1038/s41593-018-0145-X, published online 7 May 2018.

In the version of this article initially published, the annotation accompanying ref. 47 ended with "though the modulation of microglia." The first word of this phrase should have been "through." The error has been corrected in the HTML and PDF versions of the article. 